\title{
The effect of additives metal on the D.C conductivity of epoxy resin
}

\author{
Nadia A. Ali* \\ Received 12, March, 2009 \\ Accepted 23, November, 2009
}

\begin{abstract}
:
The present studies are focused on the modification of the properties of epoxy resin with different additives namely aluminum, copper by preparing of composites systems with percentage $(20 \%, 40 \%$ and $50 \%)$ of the above additives. The experimental results show that the D.C of conductivity on wt\% filler content at (293413 ) K electrical conductivity of all above composites increased with temperature for composites with filler contact and find the excellent electrical conductivity of copper and lie between $\left(2.6^{*} 10^{-10}-2.1^{*} 10^{-10}\right) \Omega . \mathrm{cm}$. The activation energy of the electrical conductivity is determined and found to decrease with increasing the filler concentration.
\end{abstract}

Key words: Electrical conductivity, epoxy resin, D.C conductivity, activation energy.

\section{Introduction:}

Conductive polymers known as composites are formulated by inclusion certain materials, which have specific properties, like carbon black, graphite and metal powders, into the bulk of a polymer used . Polymers can be made to conduct electricity intrinsically and extrinsically. Electricity is conducted through polymers by electrons and or ions. Also, there is a great need for materials having an intermediate conductivity to use in cable isolation and as flexible heating elements [1].

In addition, conductive polymers are used as electrical devices like relay switches, photoconductive polymers are used in electro imagine .Polymeric materials can provide specific micro heterogeneous reaction environments which facilities charge separation from the excited state. For these reasons, polymers had been used in direct solar conversion processes, and they helped man to understand energy transfer processes [2,3]. One of the important

applications of polymers is to use mineral fillers having a range of electrical properties to formulate composites .Polymers are typically good insulators but can be made to conduct by doping.. Epoxies are widely used in insulation, such as electrical machinery, switchgear and bushings in transformers (Wuand Tung 1995)[4]. Polymers have a very low concentration of free charge carriers, and thus are non conductive and transparent to electromagnetic radiation. Due to this reason they are not suitable for use as enclosures for electronic equipment because they cannot shield it from outside radiation. This drawback has led to the development of electrically conductive polymers such as inherently polyaniline conductive or polymers filled with conductive particles. Beyond a critical concentration of filler polymer becomes conductive [5]. This formation of a network permits the

*University of Baghdad, College of Science, Department of Physics 
movement of charge carriers of the fillers through the matrix and so the composites achieve a certain degree of electrical conductivity (Tstra and Freidrich 2003).Several fillers can be added to the insulating polymeric matrix in order to achieve different conductivity ranges [6].

Although copper is the metal that is longest used technologically by mankind and aluminum one of the shortest, in both industries a variety of melt treatments and sometimes similar technologies were investigated over time. But as for most industries a look into the technology of a non competing neighbor often is missing. Due to the noble character of copper, most elements like silicon, aluminum and iron can easily be removed from a copper melt by selective oxidation at least down to a very low concentration (activity) [7]. The polymer/metal composite material could be engineered with built-in wear resistance because the flow of polymeric material can produce autohealing effect in damaged areas on the surface during extended service. However, the development is hindered by the lack of physical insights on fatigue, which is caused by repeated cyclic loading in dynamic condition [8].

An example of copper/epoxy application is in the manufacturing of printed circuit boards. The epoxy composite serves as a dielectric layer to separate the layers of copper circuitry, while providing the necessary structural integrity of the board. For this purpose, the bonding between epoxy and copper is important. The excellent electrical conductivity of copper gives a great advantage in integrated chip (IC) manufacturing, and epoxies are often used as a packaging material along with silicones and polyimide [9]. When the polymers are applied to the metallic substrates, the interaction between the matrix and metal surface creates an inter phase, which has different chemical, physical, and mechanical properties from the bulk polymer. Metals and polymers have very different properties, when the two materials are in contact, owing to difference of their properties; a desired adhesion is often hard to be achieved. Being molecularly closely packed with strong metallic bonds, the metals have much higher cohesive energy (Ecoh) than the polymers, whose cohesion is due to weak dander Waals bonds [10].

Most widely used conductor is copper: inexpensive, abundant, high $\boldsymbol{\sigma}$, but rather soft - cannot be used in applications where mechanical strength is important. When weight is important one uses aluminum, which is half as good as $\mathrm{Cu}$ and more resistant

to corrosion. One of the best materials for electrical conduction (low resistivity) is silver, but use restricted due to high cost .Most widely used conductor is copper: inexpensive, abundant, high $\boldsymbol{\sigma}$, but rather soft cannot be used in applications where mechanical strength is important. Solid solution alloying and cold working improve strength but decrease conductivity. When weight is important one uses aluminum, which is half as good as $\mathrm{Cu}$ and more resistant to corrosion. Table (1) gives the electrical conductivity of metal [11, 12].

Table (1): The electrical conductivity of metal [11, 12].

\begin{tabular}{lc}
\hline Metal & $\begin{array}{c}\text { Electrical Conductivity } \\
{\left[(\boldsymbol{\Omega}-\boldsymbol{m})^{-\mathbf{1}}\right]}\end{array}$ \\
\hline Silver & $6.8 \times 10^{7}$ \\
Copper & $6.0 \times 10^{7}$ \\
Gold & $4.3 \times 10^{7}$ \\
Aluminum & $3.8 \times 10^{7}$ \\
Iron & $1.0 \times 10^{7}$ \\
Brass (70 Cu-30 Zn) & $1.6 \times 10^{7}$ \\
Platinum & $0.94 \times 10^{7}$ \\
Plain carbon steel & $0.6 \times 10^{7}$ \\
Stainless steel & $0.2 \times 10^{7}$ \\
\hline
\end{tabular}


The conductivity of these materials in DC electric fields where electrical conductivity $\boldsymbol{\sigma}$ is rapidly increases as then increased concentration. In this work, various epoxy resin/conductive filler composites were prepared. The filler was metal powder of copper, and aluminum. The conductive behavior of these materials was studied at temperatures varied from (293-413) K.

\section{Theory:}

\section{1-Conductivity:}

Electrical conductivity is defined as the proportional factor between the current and the electron field. Electrical conductivity of a material is a property with highest variations in values. The range observed in materials covers a range of 25 order of much magnitude [1-4].

To measure the electrical resistance of insulating materials, three electrodes cell or (guard ring electrode method) was used to study the effect of the filler addition and the temperature on volume resistivity of polymer composite[5].

Resistivity ( $\rho$ ) and DC conductivity ( $\sigma$ d.c.) values were calculated by using the following relation

Where:

$$
\rho=\mathbf{R} * \mathbf{A} / \boldsymbol{\ell} \ldots . .1
$$

$\boldsymbol{\ell}$ is the length (in units of meters)

$\boldsymbol{A}$ is the cross sectional area (in units of $\mathrm{m}^{2}$ )

$\boldsymbol{\rho}$ is the resistivity of the material (in units of $\Omega \cdot \mathrm{m}$ )

$\boldsymbol{R}$ is the resistance of the object, measured in Ohms, equivalent to $J \cdot s / \mathrm{C}^{2}$

Resistivity is a measure of the material's ability to oppose electric current. Where Conductivity was calculated by using the following formula

$\sigma_{\text {d.c }}=1 / \rho \ldots .2$
For non metallic materials, the electrical conductivity depends on temperature $\mathrm{T}$ :

$$
\sigma_{\text {d.c }}=\sigma_{0} \exp \left(-E_{\mathrm{a}} / K_{B} T\right) \ldots 3
$$

Where:

$\boldsymbol{E}_{\mathrm{a}}$ is the activation energy

$\mathbf{K}_{\mathrm{B}}$ is the Boltzmann constant

бo is the minimum electrical conductivity at $0 \mathrm{~K}$.

The electrical conductivity is a function of temperature, if the increasing of temperature causes an increasing in free change carries, and electrical conductivity increases. The rate of activation depends on the number of molecules or particles having sufficient thermal energy to reach the energy barrier and on the velocity of these molecules or atoms that can cross this energy. When the conductivity $\boldsymbol{\sigma}$ is measured at a wide of temperature, $\boldsymbol{E}_{\text {a }}$ can be calculated by plotting $\boldsymbol{\sigma}$ against absolute temperature to give a straight line show in Fig (1). [6].

The electrical conductivities of the intrinsically conducting-polymer systems now range from those typical of insulators $\left(<10^{-10} \mathrm{~S} / \mathrm{cm}\left(10^{-10} \Omega^{-1} \mathrm{~cm}^{-1}\right)\right)$ to those typical of semiconductors such as silicon $\left(\sim 10^{-5} \mathrm{~S} / \mathrm{cm}\right)$ to those greater than $10^{+4} \mathrm{~S} / \mathrm{cm}$ (nearly that of a good metal such as copper, $5 \times 10^{5} \mathrm{~S} / \mathrm{cm}$. The polymer backbone and do pant ions from new three dimensional structures. There is a rich variety of these structures, with differing structures occurring for different do pant levels and variations in the processing routes, along with a wide range of degrees of local order. For an isolated, one-dimensional metallic chain, localization of charge carriers arises for even weak disorder because of quantum interference due to backscattering of electrons. Localization effects in homogeneously disordered (partially crystalline) conducting polymers originates from one-dimensional localization in the 
disordered regions that connect the relatively ordered regions (or "crystalline islands")[10-12].

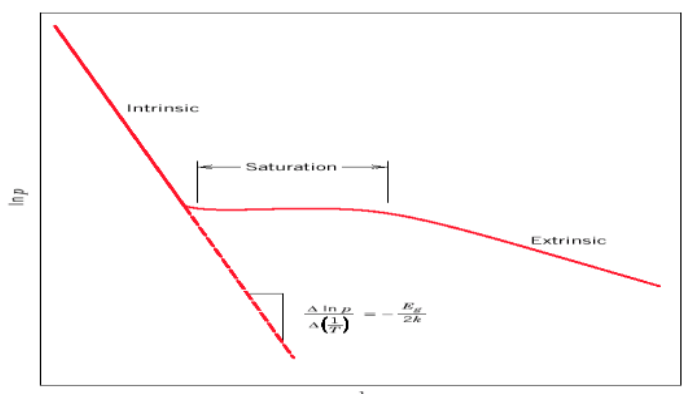

Fig (1): Illustrate the variation of In $\sigma$ vs. $1 / T$

\section{2- Activation energy:}

The values of activation energies have been calculated from the Arrhenius plots using the equation (3).

Composition effect on the electrical properties could be studied with different temperature ranges and also hopping conduction at low temperature region (293-343) $\mathrm{K}$ as well as the high temperature where band conduction occurs included. Some electrical parameters could be obtained such as the activation energy, $\Delta \mathbf{E}_{\boldsymbol{\sigma}}$, conductivity at room temperature, $\sigma_{\mathbf{R T}}$, where the activation energy have a different two values; activation energy for high conduction $\Delta \mathbf{E}_{\mathbf{1}}$, and the activation energy for low conduction, $\Delta \mathbf{E}_{\mathbf{2}}$. A polycrystalline with a negative slope is obtained. For the present composites, these plots show polycrystalline with negative slopes indicating the temperature dependence of conductivity [13].

\section{Materials and Methods:}

\section{1- Polymer:}

The material used as polymer matrix, epoxy resin type (EP Vantico CY1300) and hardener type (HY-956) in ratio $3: 1$ for curing.

\section{2- Fillers:-}

Three types of metals used in this work (copper, and aluminum) supplied by LDH chemicals Ltd (England).A weight amount of epoxy was mixed with metal powder in $(20 \%, 40 \%$ and $50 \%$ ) percentage.

\section{3- Measurement of D.C Resistivity:}

D.C volume resistivity or the corresponding conductivity of a dielectric material can be determined by several methods. According to ASTM (D257-78)recommendation [3a] for measurement of electrical resistance of insulating materials, three -electrodes cell or (guard ring electrode method) was used to study the effect of the filler addition and the temperature on volume resistivity of polymer composites .The ASTM standard states that the volume resistivitiy $\boldsymbol{\rho}$ is calculated as follows (1).

The electrodes were made of copper, and a uniform pressure on the specimen was applied by four adjusted screws. The electrical input was providing by means of stabilized D.C power supply and the output current was measured by Keithley (616). The volume resistance calculated for each reading and average $R$ has been taken. The volume conductivity measurements were performed in the temperature range (293-413) K.

\section{Result and Discussion:}

D.C conductivity was calculated using eq.(2), Fig $(2,3)$ show the dependence of D.C conductivity on wt $\%$ filler content at (293-413) K, and show that the increase in D.C conductivity of all sample with the concentration (20\%,40\%, and 50\%)of copper and aluminum filler that variation of D.C was lowest within a range of $20 \%$, and $40 \%$, when the concentration increased above $40 \%$ and reached $50 \%$, a large increased of D.C electrical conductivity in all samples was observed to increase of ionic charge carriers which might be increased due to increasing filler 
content. At low filler content (20\%) was mainly due to the polymer itself and not to the filler. This increased could be attributed to increased segmental mobility of the polymer chains near the filler particles [6]. At $50 \%$ filler concentration the increase in electrical conductivity could be ascribed to increase of ionic charge carriers which might be increased due to increasing filler content for example of copper the conductivity increased between $\left(2.6 * 10^{-11}-2.1 * 10^{-11}\right) \Omega . \mathrm{cm}[7]$. In this situation the electrical properties of filler were almost dominated, since a network may start to connect filler particles to each other and a new kinetic path may be formed. However, at the lower concentration of filler both polymer and filler ruled the electrical properties of the composite material as concluded by others. It has been observed that d.c. conductivity suddenly increases after $333 \mathrm{~K}$ in all the samples. This is because $\mathrm{Tg}$ (glass translation temperature) epoxy is around 333K, below $\mathrm{Tg}$ d.c. conductivity does not increase much, after $\mathrm{Tg}$ where epoxy comes in amorphous phase and sudden change in conductivity occurs. This is because after $\mathrm{Tg}$ free volume increases and chains start moving, which makes the movement of charge carriers easy and hence they take place in conduction process after release from traps, makes the movement of charge carriers easy and hence they take place in conduction process after release from traps [8].

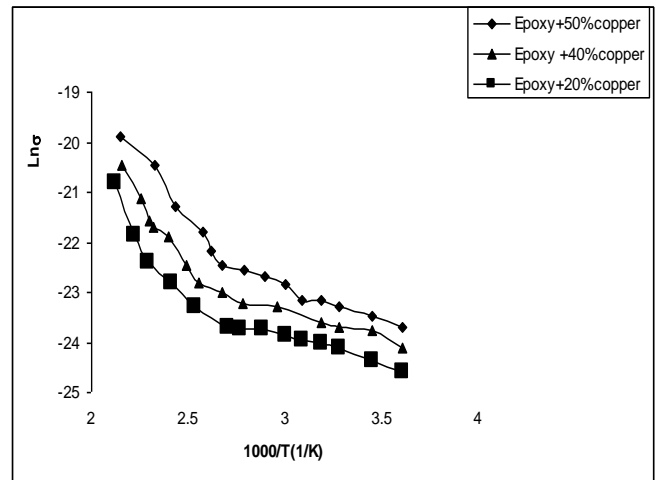

Fig(2): The variation of $\ln \sigma_{\text {d.c }}$ and $1000 / T$ for the epoxy/copper (a):20\%,(b):40\%,(c):50\%

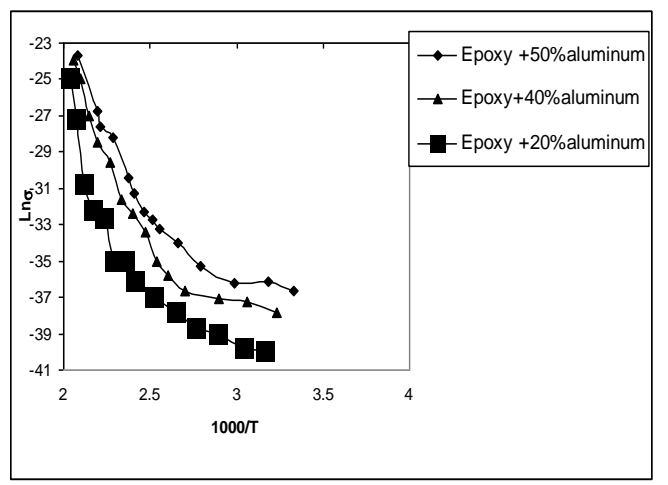

Fig(3): The variation of $\ln$ od.c and 1000/T for the epoxy/aluminum (a):20\%,(b):40\%,(c):50\%

To calculate the activation energy for the thermal activation processes Arrhinus eq.(3) has been used . Fig (4 a,b) show change the activation energy for epoxy -metal powder composites of different filler concentrations. At low temperatures the thermal activation of the conductivity was almost negligible, activation energy of thermal degrees (293-328)K except for the transition of positive energy levels localized in the energy gap and as a result of a proposed high density of localized energy levels in the energy gap[7], after increasing the temperature above $\mathrm{Tg}$ the conductivity increased strongly. So, the activation energy values were calculated for this region because that the second region (333373) $\mathrm{K}$ the result of activation energy at the granular ion by thermal emission (thermal stimulation cross-border 
movement)[8], and listed in Table(2). The activation energy values decreased with increasing of wt $\%$ filler content of epoxy-metal composition. The low activation energy for epoxy -copper between (0.17-0.11) e.V should be to the electronic conduction mechanism which was resulted fro a new kinetic path formation in polymer matrix. It has been reported that for these types of composites ionic, electronic and even mixed conducting process are possible [1].This value is compared with epoxy pure and that activation energy of $1.33 \mathrm{eV}[2]$.

Table (2): Values of $\sigma_{\text {d.c }}$ and activation energy of polymer/metal.

\begin{tabular}{|c|c|c|c|}
\hline Additives & $\boldsymbol{\sigma}_{\text {d.c }}(\mathrm{R} . \mathrm{T})$ & $\mathrm{E}_{\mathrm{a} 1(\mathrm{e} . \mathrm{v})}$ & $\mathrm{E}_{\mathrm{a} 2 \text { (e.v) }}$ \\
\hline $20 \%$ Copper & $2.6 * 10^{-10}$ & 0.26 & 0.17 \\
\hline $40 \%$ Copper & $2.2 * 10^{-10}$ & 0.19 & 0.16 \\
\hline $50 \%$ Copper & $2.1 * 10^{-10}$ & 0.06 & 0.11 \\
\hline $20 \%$ Aluminum & $6.7 * 10^{-10}$ & 0.27 & 0.19 \\
\hline $40 \%$ Aluminum & $6.4 * 10^{-10}$ & 0.21 & 0.18 \\
\hline $50 \%$ Aluminum & $6.1 * 10^{-10}$ & 0.09 & 0.15 \\
\hline
\end{tabular}

This decrease of activation energy with increase of metal content is similar to the activation energy calculated for filled blends (Anatoly et al 1998), which has been explained by the formation of border layer at the interface of the filled polymer [9]. The activation energy calculated with an Arrhenius equation for one sample was $0.06 \mathrm{e.V}$ (at filler 50\%copper) and this was mainly due to conduction electronic. Copper is a superb conductor of heat, much better than other kitchen metals. Only silver is a better metal, but even silver is only about $7 \%$ better than copper. High Conductivity Copper, 99.9\% pure. This grade of Copper was developed for high conductivity, both electrical and thermal [14].
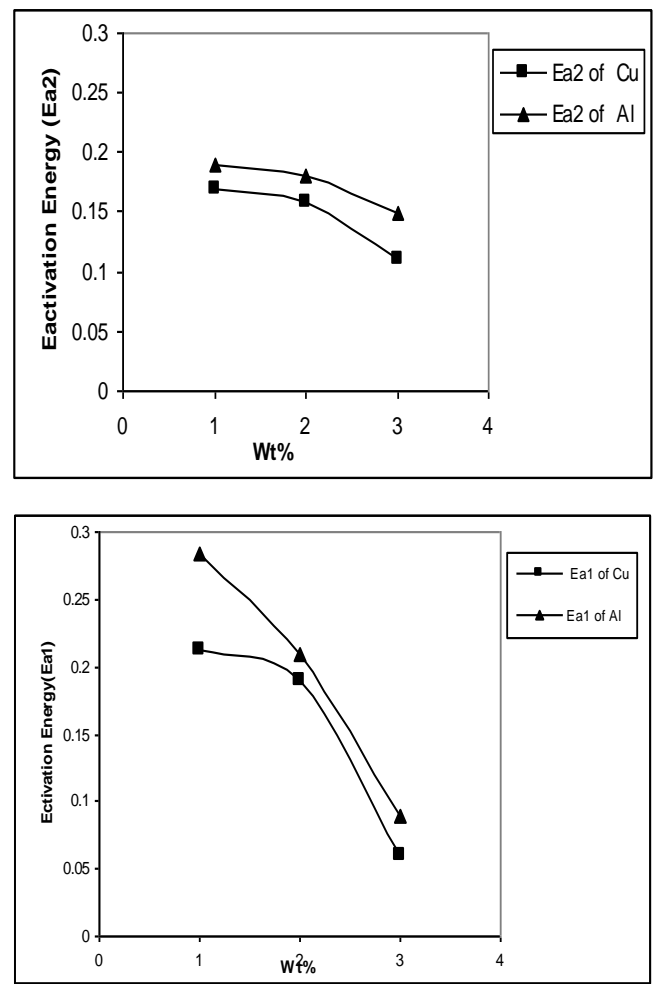

Fig (4): Activation Energy (Ea1, Ea2) for D.C electrical conductivity for all samples.

\section{Conclusion:}

1-The electrical conductivity of all above composites increased with increased temperature for composites epoxy and filler (aluminum, and copper) contact.

2-The best conductivity of Copper was developed for high conductivity, both electrical and thermal.

3-The activation energy decreased with increase of metal content and increased temperature.

\section{Reference:}

1-Tsangaris G.M., and Kazilas M.C., 2008" Conductivity and percolation in epoxy resin/conductive filler composites" J. Phys. D: Appl. Phys., 16(21): 41-45.

2- Elima Z. M., Zihlif A.M. and Ragosta G., 2008" Study of a.c electrical properties of aluminumepoxy composites" J. Phys. D: Appl. Phys., 41 (16):7-9.

3- Kuo-Chung Ch , Chien-Ming L., Sea-Fue W., Shun-Tian L. and 
Chang-Fa Y., 2007, Dielectric properties of epoxy resin-barium titanate composites at high frequency", Science Direct ,61(5) : 757-760

4-Vishal S., Kulkarni A.R., and Rama Mohan T. R., 2003,"Dielectric properties of aluminum-epoxy composites" Journal of applied polymer science, 90(13):3602-3608

5- Navin CH. and Archana N., 2008" Investigations on d.c. conductivity behavior of milled carbon fiber reinforced epoxy graded composites" Bull. Mater. Sci., 31(4):665-668.

6. KawamotoY. , Nagura N. , and Tsuchihashi S., 2006 "Dc Conductivity of Ge-S-Ag and AsS-Ag Glasses" Journal of American Ceramic Society, 57(11): 489 - 491 .

7- Sindhu S., Anantharaman M. R., Thampi B. , Malini K. A. and Kurian P. 2002" Evaluation of a.c. conductivity of rubber ferrite composites from dielectric measurements" Bull. Mater. Sci., 25 (7): 599-607.

8-Sankara S., Arayanan Potty N. ,and Abdul Khdar M. ,2000" Dielectric properties of nanophase $\mathrm{Ag} 2 \mathrm{HgI} 4$ and $\mathrm{Ag} 2 \mathrm{HgI} 4-\mathrm{Al} 2 \mathrm{O} 3$ nanocomposites" Bull. Mater. Sci., 23(5): 361-367.

9-Tsangaris G.M., and Kazilas A.S., 2002" Conductivity and percolation in epoxy resin/conductive filler composites" Materials Science and Technology, 18 (5) :226-230.

10- Kohlman R.S. and Epstein A.J. , 1997" Insulator-Metal Transition and Metallic State of Conducting Polymers", Handbook of Conducting Polymers, Second Edition, edited by T.A. Skotheim, R.L. Elsenbaumer, and J.R. Reynolds (Marcel Dekker, Inc.), Ch. 3:85-121

11-Yun Z., Kim Chonung G. W., and Ping Wei, P. J., 2006" Improving the Dielectric Properties of Polymers by Incorporating Nanoparticles" Applied Science and Manufacturing Composites, Part A: ,37(3 ):1545-1553.

12- Chand N., and Jain D. , 2004 "Evaluation of a.c. conductivity behaviour of graphite filled polysulphide modified epoxy composites'" Bull. Mater. Sci., 27(3): 227-233.

13-Patidar D. , Jain N. , Saxena N.S , Kananbala S. , and Sharma T.P, 2006"Electrical Properties of CdS/Polyaniline Heterojunction" Brazilian Journal of Physics, 36(4):210-1212.

14-Singh V., Kulkarnia R., and Rama M.R., 2003" Dielectric properties of aluminum-epoxy composites "Journal of applied polymer science, 90(8):3602-3608.

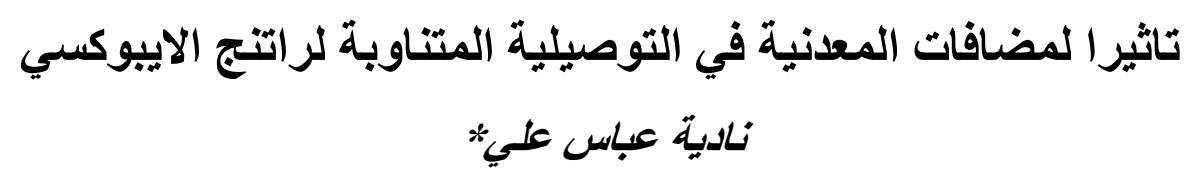

*جامعة بغداد ,كلية العلوم ,قسم الفيزياء

ركزت هذه الدر اسة على در اسة خصـائص ر اتنج الاييوكسي ومضـافات مختلفة مـن المعـادن (نحساس, و المنيوم)

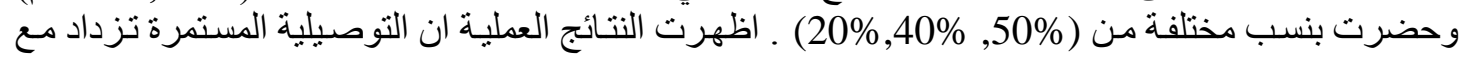

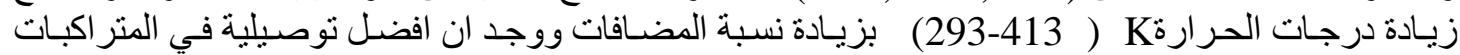
المتكونة من البوليمر و النحاس تقع بين 2.cm تتناقص مع زيادة نسبة المضافات للمتر اكبات كلها . 